\title{
Reassessment of Lymphocytic Atypia in the Diagnosis of Mycosis Fungoides
}

Y. Albert Yeh, M.D., Ph.D., Amy R. Hudson, M.D., Victor G. Prieto, M.D., Ph.D., Christopher R. Shea, M.D., Bruce R. Smoller, M.D.

Departments of Pathology (YAY, ARH, BRS) and Dermatology (BRS), University of Arkansas for Medical Sciences, Little Rock, Arkansas; and Department of Pathology (VGP, CRS), Duke University Medical

Center, Durham, North Carolina

The manifestations of mycosis fungoides in its early stage may mimic clinically and histologically those of many benign inflammatory dermatoses. Therefore, the diagnosis of mycosis fungoides remains a major challenge for dermatologists and dermatopathologists. For many years, it has been proposed that atypical lymphocytes within the epidermis constitute one of the diagnostic features in mycosis fungoides. Presence of dermal atypical lymphocytes remains controversial as a diagnostic criterion. We reassessed the feasibility of applying lymphocytic atypia within epidermis and dermis as diagnostic criteria discriminating between mycosis fungoides and spongiotic dermatitis. Thirty cases of mycosis fungoides and 30 cases of spongiotic dermatitis were retrieved from archival hematoxylin and eosin-stained histologic sections. Punch biopsy sections were examined by light microscopy; epidermal and dermal lymphocytes were photographed at $1000 \times$ (oil immersion). A total of 92 ektachrome slides (35 mM) were developed, coded, and ordered randomly. For each slide, cells were interpreted as typical or atypical lymphocytes by seven pathologists. Atypical epidermal lymphocytes were judged to be present in $9 \pm 2$ out of $16(56 \%)$ cases of mycosis fungoides photographed as compared with $8 \pm 3$ out of $16(50 \%)$ in spongiotic dermatitis. Dermal lymphocytic atypia was thought to be present in $14 \pm 6$ out of $30(47 \%)$ patients with mycosis fungoides. Thirteen \pm 6 out of 30 (43\%) patients with non-mycosis fungoides also displayed dermal lymphocytic atypia. No statistical significance was observed in these comparisons $(t$ test, $P>.05)$. Furthermore, atypia of lymphocytes was deemed to

Copyright () 2001 by The United States and Canadian Academy of Pathology, Inc.

VOL. 14, NO. 4, P. 285, 2001 Printed in the U.S.A

Date of acceptance: October 24, 2000.

Address reprint requests to: Bruce R. Smoller, M.D., University of Arkansas for Medical Sciences, Department of Pathology, 4301 West Markham Street, Slot 517, Little Rock, AR 72205; e-mail: SmollerBruceR@exchange.UAMS.edu; fax: 501-603-1479. be present in $41,38,59,70,23,47$, and 40 out of 92 slides examined by the investigators, suggesting that observer variation is a very significant factor in our present study. We conclude that it is not possible to distinguish mycosis fungoides from spongiotic dermatitis merely based on lymphocytic atypia within epidermis or dermis.

KEY WORDS: Mycosis fungoides, Spongiotic dermatitis, Atypical lymphocytes.

Mod Pathol 2001;14(4):285-288

Mycosis fungoides (MF), one form of cutaneous T-cell lymphoma, first was described by Alibert in 1806 (1). The onset of the disease is insidious, and its clinical course is usually relentless. The histologic features of MF initially were described in the early 20th century (2). Fraser et al. (3) depicted the differential diagnosis of MF and psoriasis. MF in its early stage may mimic psoriasiform and lichenoid inflammatory dermatoses both clinically and histologically; therefore, the diagnosis remains a major challenge for dermatologists and dermatopathologists. Investigators have proposed histologic criteria distinguishing MF from non-MF (4-8). Parameters suggesting a diagnosis of MF included atypical intraepidermal lymphocytes surrounded by halos, Pautrier's microabscess, exocytosis, disproportionate epidermotropism, epidermal lymphocytes larger than dermal lymphocytes, hyperconvoluted intraepidermal lymphocytes, and lymphocytes aligned within the basal layer (4-9). Presence or absence of atypical lymphocytes within dermis remains controversial. It was the purpose of this present study to reassess the significance of atypical lymphocytes within epidermis and dermis and the feasibility of applying these parameters in discriminating between MF and non-MF.

\section{MATERIALS AND METHODS}

Routine hematoxylin and eosin-stained histologic sections from 30 cases of patch-stage MF and 
30 cases of spongiotic dermatitis were retrieved from slide archives at the University of Arkansas for Medical Sciences, Department of Pathology. A diagnosis of MF was rendered based on clinical suspicion of MF, histologic features suggestive of the disease, immunophenotypic analysis of cutaneous lymphocytes, and a minimum of 3-year follow-up with a diagnostic clinical course. Cases of spongiotic dermatitis were selected based on a typical clinical picture suggestive of spongiotic dermatitis, pathologic findings without conclusive evidence of $\mathrm{MF}$, and clinical follow-up for a minimum of at least 3 years. Punch biopsies of skin were examined by light microscopy; the most atypical lymphocytes within the epidermis and the dermis were photographed at $1000 \times$ oil immersion by one of us (AY). Atypia for the sake of photographic selection was defined as enlarged lymphocytes with hyperchromatic nuclei and nuclear infolding. Histologic sections displaying large atypical lymphocytes with pale vesicular nuclei and prominent nucleoli that predominate in advanced stages of MF were excluded from this study and were not found in significant numbers in any of the cases studied. A total of 92 ektachrome slides (35 mM) were used. These slides included 32 slides of epidermal lymphocytes from 16 of the 30 cases of MF and 16 of the 30 cases of spongiotic dermatitis; 60 slides of dermal lymphocytes from all cases of MF and all cases of spongiotic dermatitis also were evaluated. Ektachrome slides were coded, randomly mixed, and circulated to a panel of seven expert pathologists who had no knowledge of the clinical or histologic diagnoses. Lymphocytes were interpreted as either typical or atypical based on each reviewer's own concept of cytologic atypia. There was no prior training session and no discussion about criteria to use for the designation of atypia. Numbers of slides judged as depicting atypical lymphocytes within epidermis and dermis from MF were averaged, and the mean was compared with that of spongiotic dermatitis. Student's $t$ test was applied, and values yielding a probability of $P<.05$ were considered statistically significant.

\section{RESULTS}

Figure 1 shows a representative slide of what the majority of observers $(4 / 7,57 \%)$ called atypical epidermal lymphocytes. They are characterized by slightly enlarged, hyperchromatic, and hyperconvoluted nuclei. These cells are from a patient with MF. These cytologic features were in contrast to those thought by most observers to represent reactive lymphocytes, as are seen in Figure 2. These cells are from a patient with spongiotic dermatitis.

Atypical epidermal lymphocytes were believed by the majority of observers to be present in $9 \pm 2$ out

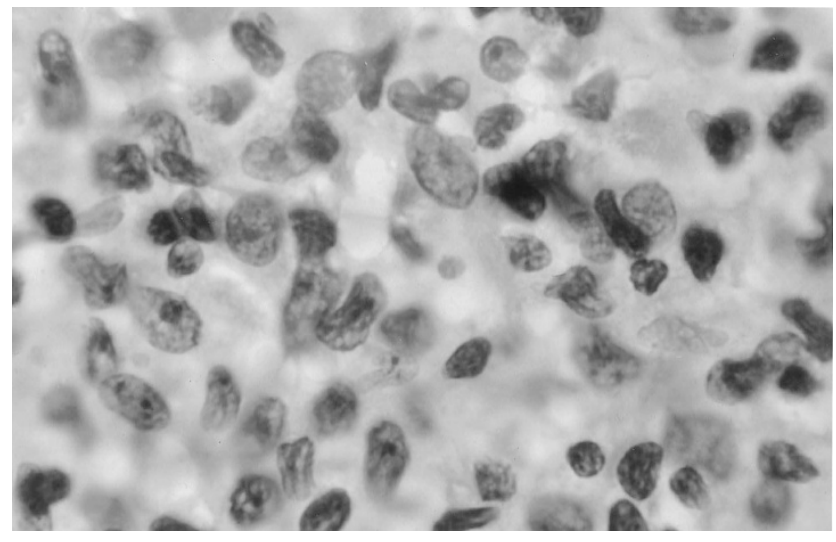

FIGURE 1. Slightly enlarged, hyperchromatic, hyperconvoluted lymphocytes within epidermis (mycosis fungoides, 1000×).

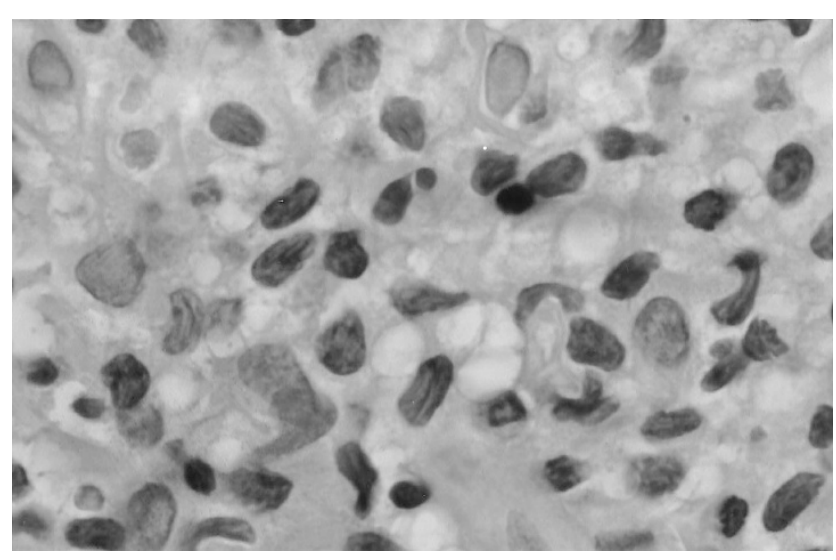

FIGURE 2. Benign reactive lymphocytes within dermis (spongiotic dermatitis, $1000 \times)$.

of $16(56 \%)$ cases of MF as compared with $8 \pm 3$ out of $16(50 \%)$ in spongiotic dermatitis (Table 1). Lymphocytic atypia within the dermis was called by the majority of observers in $14 \pm 6$ out of $30(47 \%)$ patients with MF. Thirteen \pm 6 out of 30 (43\%) patients with spongiotic dermatitis also displayed dermal lymphocytic atypia according to the observers (Table 1). There was no statistical significance observed between MF and spongiotic dermatitis cases $(P>.1)$ (Table 1). Table 2 shows the interobserver variation. Lymphocytic atypia was considered to be present in 41 (45\%), 38 (41\%), 59 (64\%), 70 (76\%), 23 (25\%), 47 (51\%), and 40 (43\%) out of 92 slides, respectively, by each of the seven observers. Assessment of lymphocytes as atypical ranged from $25 \%$ of cases to $76 \%$ of cases (Table 2). Complete agreement (7/7 of observers) on lymphocytic atypia could be reached in only $9 \%$ of cases. Out of 92 slides, $6 / 7$ agreed in $15 \%, 5 / 7$ agreed in $34 \%$, and $4 / 7$ agreed in $30 \%$. None of the observers thought there was atypia in $12 \%$ of cases.

\section{DISCUSSION}

MF is a very difficult diagnosis to make in its initial stages for both clinicians and pathologists. A 
TABLE 1. Typical or Atypical Lymphocytes Observed in Mycosis Fungoides versus Spongiotic Dermatitis

\begin{tabular}{|c|c|c|c|c|c|}
\hline \multirow{2}{*}{$\begin{array}{c}\text { Epidermis versus } \\
\text { Dermis }\end{array}$} & \multirow{2}{*}{$\begin{array}{l}\text { Dermato- } \\
\text { pathologist }\end{array}$} & \multicolumn{2}{|c|}{$\begin{array}{c}\text { Mycosis } \\
\text { Fungoides }\end{array}$} & \multicolumn{2}{|c|}{$\begin{array}{l}\text { Spongiotic } \\
\text { Dermatitis }\end{array}$} \\
\hline & & Typical & Atypical & Typical & Atypical \\
\hline \multirow[t]{8}{*}{ Epidermis } & 1 & 6 & 10 & 8 & 8 \\
\hline & 2 & 8 & 8 & 9 & 7 \\
\hline & 3 & 8 & 8 & 7 & 9 \\
\hline & 4 & 3 & 13 & 4 & 12 \\
\hline & 5 & 10 & 6 & 13 & 3 \\
\hline & 6 & 6 & 10 & 6 & 10 \\
\hline & 7 & 8 & 8 & 9 & 7 \\
\hline & Mean $\pm S D$ & $7 \pm 2$ & $9 \pm 2$ & $8 \pm 3$ & $8 \pm 3$ \\
\hline \multirow[t]{8}{*}{ Dermis } & 1 & 18 & 12 & 21 & 9 \\
\hline & 2 & 15 & 15 & 23 & 7 \\
\hline & 3 & 10 & 20 & 10 & 20 \\
\hline & 4 & 5 & 25 & 11 & 19 \\
\hline & 5 & 22 & 8 & 24 & 6 \\
\hline & 6 & 22 & 8 & 11 & 19 \\
\hline & 7 & 19 & 11 & 16 & 14 \\
\hline & Mean $\pm \mathrm{SD}$ & $16 \pm 6$ & $14 \pm 6$ & $17 \pm 6$ & $13 \pm 6$ \\
\hline
\end{tabular}

SD, standard deviation.

large number of studies have attempted to put forth histologic criteria that can be used to reliably distinguish benign spongiotic processes from this frequently indolent cutaneous T-cell lymphoma. In many of these studies, cytologic atypia, as defined by the presence of lymphocytes with hyperconvoluted (or cerebriform) and hyperchromatic nuclei, has been proposed to be a nearly constant finding (4-8). In some studies, emphasis was placed on the specificity of identifying these cells within the epidermis as opposed to within the dermis. Some authors have advocated the use of cytologic preparations including fine needle aspirations, biopsy imprints, and Wright-stained cytologic smears to detect these "atypical" cells (10-12).

In an earlier study, some of the present authors called into the question the specificity of these hyperconvoluted cells (9). In their study, hyperconvoluted and hyperchromatic lymphocytes were detected in the great majority of cases of MF. However, the same observers thought the same types of cells were present within the dermis of many reactive histologic simulants.

In many of the previous studies evaluating the usefulness of histologic criteria in arriving at a diagnosis of patch-stage MF, an unavoidable archi-

TABLE 2. Interobserver Variation

\begin{tabular}{ccc}
\hline Pathologist & $\begin{array}{c}\text { Typical Lymphocytes } \\
\text { (\% of Slides) }\end{array}$ & $\begin{array}{c}\text { Atypical Lymphocytes } \\
\text { (\% of Slides) }\end{array}$ \\
\hline 1 & 55 & 45 \\
2 & 59 & 41 \\
3 & 36 & 64 \\
4 & 24 & 76 \\
5 & 75 & 25 \\
6 & 49 & 51 \\
7 & 57 & 43 \\
\hline
\end{tabular}

tectural bias was introduced into the evaluation of cytologic criteria. Histologic examiners evaluated microscopic sections by evaluating a range of criteria that placed the "atypical" into a context. It is difficult, if not impossible, to remain objective when assessing subtle changes of hyperchromasia and hyperconvolution while examining lymphocytes that are present within a band-like infiltrate underlying a psoriasiform epidermis with little spongiosis and abundant epidermotropism. In the present study, we attempted to eliminate this architectural bias by presenting pathologists with very high magnification photomicrographs of lymphocytes devoid of essentially all architectural clues as to location or other changes within the biopsy specimen. Further, as individual lymphocytes were evaluated, only the cytologic features could be assured. Groups or clusters of lymphocytes with similar cytologic structures were not available for us to interpret. In this manner, we believe we were able to evaluate the sole criterion of "lymphocyte atypia" as a predictor of MF. Our data demonstrate conclusively that none of the seven reviewers was able to reliably distinguish MF from spongiotic dermatitis in a majority of cases.

In addition, the results also demonstrate that "atypia is in the eyes of the beholder" in the evaluation of lymphocyte atypia within the dermis. The pathologists involved in this study demonstrated very little ability to agree on atypia without prior training as a group. In only $9 \%$ of cases did all seven observers concur that a given lymphocyte demonstrated cytologic atypia. In approximately $30 \%$ of the lymphocytes examined, there was absolutely no agreement, with $4 / 7$ calling the cells "atypical" and 3/7 suggesting that the cells were "not atypical." The pathologists had a wide range of practice experiences, and although some of them had trained in the same institutions, they each had taken a unique career pathway and had not previously examined this question together. This is the most salient feature of our findings. Although it is possible that with a period of consensus training these same pathologists might agree on a set of criteria that constitute a diagnosis of atypia, this would not represent the situation in the real world. Pathologists are making a diagnosis of MF based, in part, on a recognition of lymphocyte atypia that is not reproducible.

It should be noted that according to the study design, the assumption was made that the lymphocytes within the cases of MF were, in fact, the neoplastic infiltrates. As is well known, many of the lymphocytes within the dermis in early cases of MF are not necessarily neoplastic, but may represent immunologically reactive cells in the host. Thus, it remains possible that at least some of the cells examined by the panel of experts from cases of MF 
may have represented reactive as opposed to neoplastic $T$ cells. Nonetheless, this does not detract from our finding that trained pathologists were unable to concur on lymphocyte atypia.

In summary, cytologic atypia does not appear to be useful as a sole criterion for arriving at a diagnosis of MF. Highly skilled microscopists were unable to agree on which cells were atypical and, further, were unable to predict whether or not a patient had MF based on the examination of lymphocytes in tissue sections.

Acknowledgments: We thank Robin Vollmer, M.D., Susan Eliason, M.D., and Steven Ruhoy, M.D., for their assistance in this cytomorphologic study.

\section{REFERENCES}

1. Alibert JL. Description des Maladies de la Peau: Observees l'Hospital St. Louis et Exposition des Meilleurs Methods Suiview pour leur Traitement. Paris. In: Barrois l'aine et Fils, 1806.

2. Galloway J, MacLeod JMH. Mycosis fungoides. An examination of three cases. Br J Dermatol 1900;12:153-87.

3. Fraser JF. The pathology of mycosis fungoides. J Cutan Dis 1917;35:793-828.
4. Lefeber WP, Robinson JK, Clendenning WE, Dunn JL, Colton T. Attempts to enhance light microscopic diagnosis of cutaneous T-cell lymphoma (mycosis fungoides). Arch Dermatol 1981;117:408-11.

5. Nickoloff BJ. Light-microscopic assessment of 100 patients with patch/plaque-stage mycosis fungoides. Am J Dermatopathol 1988;10:469-77.

6. Sanchez JL, Ackerman AB. The patch stage of mycosis fungoides. Criteria for histologic diagnosis. Am J Dermatopathol 1979;1:5-26.

7. Smith NP. Histologic criteria for early diagnosis of cutaneous T-cell lymphoma. Dermatol Clin 1994;12:315-22.

8. Shapiro PE, Pinto FJ. The histologic spectrum of mycosis fungoides/Sezary syndrome (cutaneous T-cell lymphoma). A review of 222 biopsies, including newly described patterns and the earliest pathologic changes. Am J Surg Pathol 1994; 18:645-7.

9. Smoller BR, Bishop K, Glusac E, Kim YH, Hendrickson M. Reassessment of histologic parameters in the diagnosis of mycosis fungoides. Am J Surg Pathol 1995;19:1423-30.

10. Montgomery H, Pease G. Cutaneous smears as a diagnostic aid in mycosis fungoides and other lymphoblastomas. Acta Derm Venereol 1957;2:103-11.

11. Wilson GT. Cutaneous smears: a diagnostic aid in certain malignant lesions of the skin. J Invest Dermatol 1954;22:17387.

12. Rosen SE, Koprowska I, Vonderheid EC. Skin imprint cytology in the diagnosis of cutaneous T-cell lymphomas. A preliminary report. Acta Cytol 1982;26:819-22. 